\title{
Energy-saving Technologies and Research I Using Neural Network Models
}

\author{
Sergiy V. Kovalevskyy, Ekaterina A. Zavgorodnyaya \\ Donbas State Engineering Academy, Faculty of integrated technology and equipment, Kramatorsk, Ukraine \\ Email address: \\ kovalevskii@i.ua (S. V. Kovalevskyy), tiup@dgma/donetsk.ua (E.A.Zavgorodnaya)
}

\section{To cite this article:}

Sergiy V. Kovalevskiy, Ekaterina A. Zavgorodnyaya. Energy-saving Technologies and Research I Using Neural Network Models. American Journal of Neural Networks and Applications. Vol. 1, No. 1, 2015, pp. 23-28. doi: 10.11648/j.ajnna.20150101.13

\begin{abstract}
In article he results of researches in different aspects of surface treatment are submitted. New type of the process of stabilization of residual pressure is considered. Also new ways of hardening of details are investigated and compared. The problem of new tools and the question of its more effective using are considered. The way of neural network modeling for data processing was used for in all experiments.
\end{abstract}

Key words: Surface-active Substance, Residual Stress, Electropulse Processing, Burnishing, Neural Network, Hardening, Self-propagating High-temperature Synthesis, Nonresharpened Replaceable Many-sided Plate (Nrp), Cutting Tool

\section{Introduction}

In the conditions of market economy the further development of mechanical engineering is impossible without economy material, power and a manpower, an economical expenditure of metal, fuel, the electric power. One of perspective directions in economy material and power resources is creation of less power-intensive technological processes at the expense of wide application of progressive and perspective technologies of processing of metals. Also the question of choose of tools is very difficult. Now, about $70 \%$ of the metal-cutting tool make of a firm alloy of various marks. Thus, it turns out, that to a waste there is a large quantity of a firm alloy, and it basically consists of rare and expensive materials (carbides of tungsten, the titan, etc.). Certainly, the worn out plates go to processing, but it is difficult enough and expensive process.

\section{Surface-active Substances as the Method of Stabilization of Residual Pressure}

\subsection{The Theory of Residual Stresses}

One of the factors influencing on quality of machinebuilding production is technological residual pressure which often makes defining impact on operational properties of details of cars and demands on technological management. Thermal processing as the basic method of removal of residual pressure is a power-intensive technological process that demands big floor spaces and, besides, is not harmless process [1]. The specified lacks it is deprived such method of removal of residual pressure, as drawing on the processed surface of a detail of surface-active substances.

Such substances which are adsorbed on an interface of phases are called as surface-active (SAS) and reduce superfluous superficial free energy. As a rule, have a defile structure of molecules, that is contain of fragments of molecules possessing with hydrophilous and waterproof properties [1].

It is known, that action of technological residual pressure is shown at various stages of life cycle of a product: first, after machining process; secondly, in the course of assemblage of details in a finished article; thirdly, while in service products, or performance of the office appointment by it. A problem of the given researches was to show, that at the first stage it is possible to apply to the problem decision having fallen. Also it is possible to make an assumption about their application and at the second stage.

\subsection{The Experimental Researches}

For problem studying the experimental researches which purpose was to reveal the influence of SAS on change of residual pressure in metal were made. The method of peakfrequency characteristics has been used. Measurements of 
resonant frequencies and amplitudes were carried out.

The results were affected by variable factors: various on a structure and a chemical compound of SAS; time of influence of SAS for experimental samples after its drawing; an arrow of a deflection of experimental samples; various quality of a surface of experimental samples (roughness parameter).

The plates in length of $110 \mathrm{~mm}$, by width $20 \mathrm{~mm}$, thickness of $1 \mathrm{~mm}$ from materials were used: « Steel $45 »$, « Steel 12X18H10T», «Steel 3» as samples. On each plate from both parties were pasted a piezosensor.

For influence on samples surface-active substances were used different SAS.

Experimental installation consists of an adjusting design, an oscillograph and the generator of sound frequencies. For increase of cleanliness of experiment the computerization of process with use of payment ACP (analogue-digitizer), the computer and programs Power Graph has been executed.

The received experimental data have been analysed with the help of neural network net processing. For reception of mathematical models of dependence of resonant frequencies from a kind of SAS, an arrow of a deflection of samples and time of its influence the neural network in program NeuroPro has been created and trained, the verbal description of mathematical models is received. Graphic dependences of network resonant frequencies on time of drawing of SAS on a surface of samples are constructed and analyzed on the basis of the received models.

\section{Research of Technological Possibilities of the Combined Methods of Hardening of Details of Machines}

\subsection{The Processing of Surfaces by the Concentrated Streams}

The primary goals and simultaneously problems of modern mechanical engineering is wear resistance increase, and also reliability and durability, details of machines. The

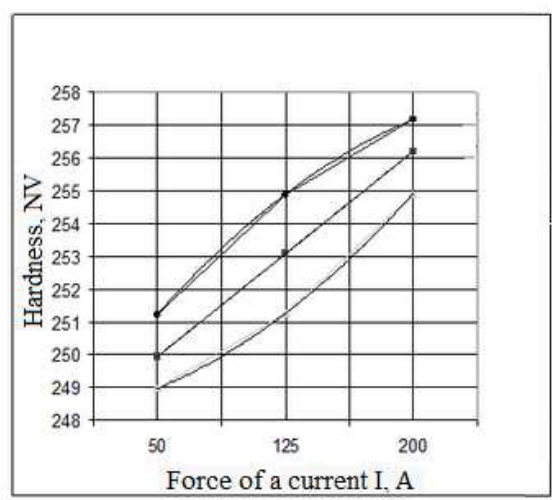

a) most actual and effective along with traditionally applied technologies are new or improved technologies [3]. To such new technological processes carry the pulse technology capable repeatedly to raise labor productivity, efficiency of use of resources, and also to decrease the material capacity and power consumption of manufacture.

Essence of these methods is processing of surfaces by the concentrated streams of energy which provide the raised intensity of technological influence on a material, the powerful energy sources based on use. Pulse methods have caused genuine interest of many researchers and experimenters and their researches are actual and till today [2].

Now possibility of a combination special turning and electromechanical burnishing, applying a roller instead of a cutter with a firm-alloyed plate, and using a pulse current, allows to receive a version of electromechanical hardening $(\mathrm{EMH})$ - electromechanical rough turning (EMRT) with use of a pulse current [3].

\subsection{The Experiments}

Experiments were made on preparations from a steel 45 and $50 Г 2$, serially established on a semi-automatic turret lathe where to preparation and the tool (cutter) at the expense of the generator of pulse currents the pulse current moved and regulated. Processing was made as at usual roughworking. Processing modes (except changeable factors) stole up experimentally.

Changeable factors:

- force of a current 50 .. 200 and;

- frequency of pulse currents $300 \ldots 700 \mathrm{~Hz}$.

Influence of changeable factors on indicators of hardness and roughness of the strengthened layer is investigated.

\subsection{The Data Processing and Results}

The Processing of experimental data were made by means of a method of neural network net modelling. It has been defined a functional dependences and schedules (fig. 1, 2) are constructed.

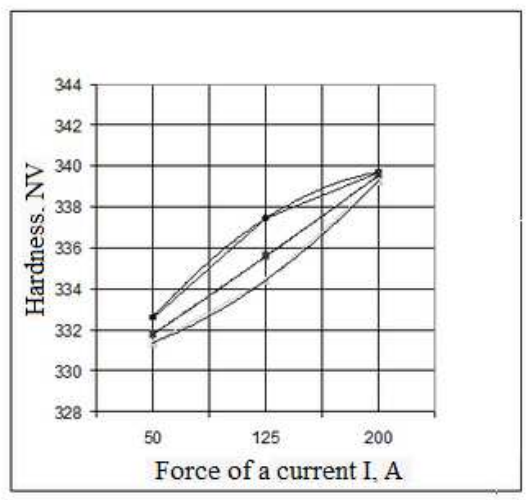

b)

Figure 1. Dependence of hardness on force of a current for a steel 45 and $50 \Gamma 2$ for $f_{1}=300, f_{2}=500, f_{3}=700$. 


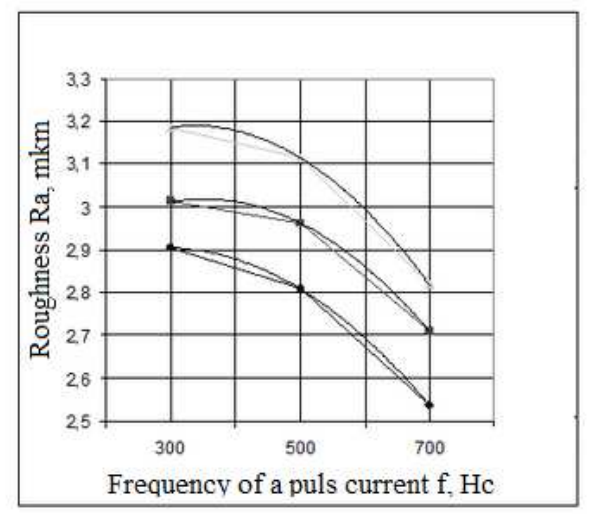

a)

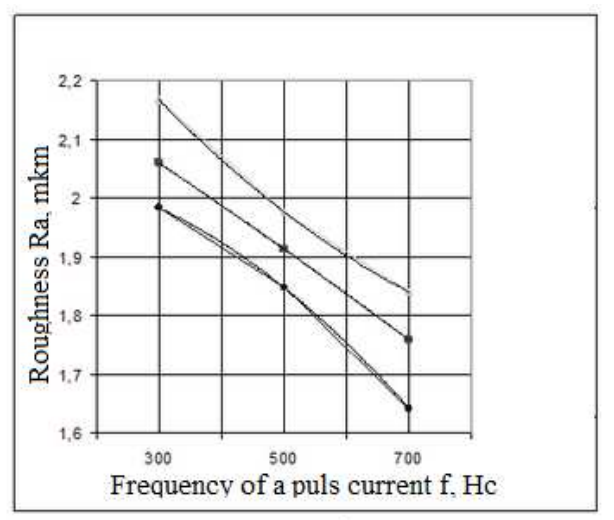

b)

Figure 2. Dependence of a roughness on frequency of a pulse current for a steel 45 and $50 \Gamma 2$ for $I_{1}=50, I_{2}=125, I_{3}=200$.

The received results show, that with increase in force of a current hardness on steels 45 and $50 Г 2$ increases non-linearly. With the increase of frequency of a pulse current of value of a roughness non-linearly decreases.

\section{Electropulse Burnishing as the Harmless Way of Hardening of Details of Machines}

\subsection{The Electropulse Burnishing}

The electropulse burnishing instead of roller burnishing at processing of samples from the tempered steel has such advantage as high durability steel characteristics that do not allow to deform substantially microroughness and to strengthen a blanket during the rolling under the influence of the warmth allocated in a point of contact of indenter with a detail, the secondary training raise the microhardness of a surface, melting and deformation of microroughnesses. That leads to formation of more wearproof surfaces [4].

Besides, at identical modes of EMP (electro mechanical processing) depth of the strengthened layer above at smoothing by the tool with motionless. As numerous experiences show, the details strengthened by the tool with motionless fastening in comparison with details, strengthened by the rotating tool, possess of more endurance ability and wear resistance.

Traditionally for electropulse burnishing spherical indenter is used, rigidly fixed roller tool or plates from a firm alloy [5]. The plate and a roller can settle down in vertical, horizontal planes, and sometimes and under some corner to a detail axis, as in our case. A holder with vertical fastening of a plate it is convenient to apply at EMP step shaft; such design of holder allows to use some times a contact surface of a plate, from time to time displacing it concerning an axis of the centers of the machine tool. A holder with a roller plate will give the chance to turn periodically a roller in process of deterioration of a contact surface and thus in tens times to increase the general firmness of the tool. Spherical indenter allows to create high efforts of stressing in a zone of contact to preparation for the account of the small area of a stain of contact.

\subsection{The Equipment}

For realization of process electropulse burnishing as an indenter a bilateral tangential plate for rough turning LNMX $2210 \mathrm{R} / \mathrm{L}-\mathrm{HT}$ has been used. It has helicoid cutting edge for work with the big depth of cutting on high giving (fig. 3).

The process of electropulse burnishing was carried out by the rounded off sides of plate LNMX 2210. R/L-HT which do not participate directly in the course of tangential turning and after deterioration of a plate on turning operation remain not touched.

As it has been mentioned above the plate in a holder settles down under a corner of 45 degrees concerning a cutting plane (fig. 4). The print of contact to preparation will have the ellipse form, unlike burnishing with a spherical indenter or a ball. These changes will allow to observe behind the received geometry of microroughnesses of a surface which, in turn, will lead to change of microhardness of a processed detail.

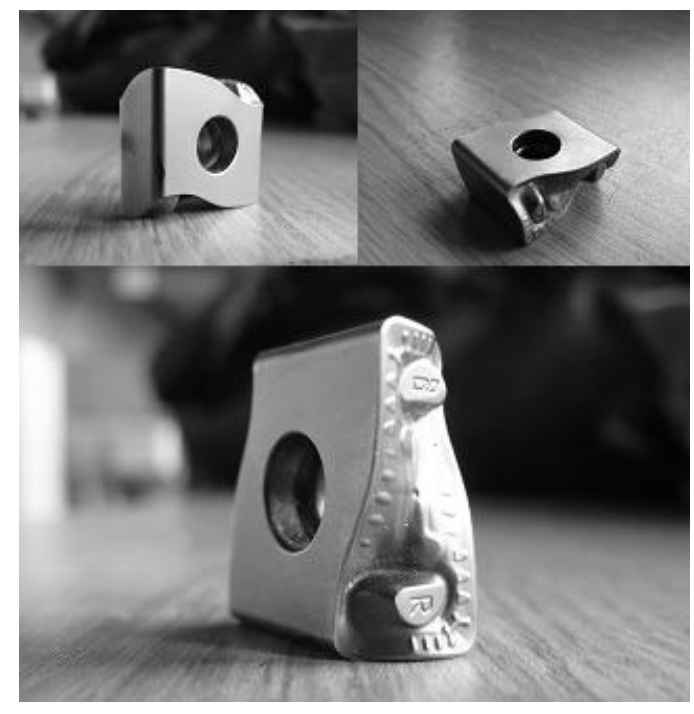

Figure 3. Plate LNMX 2210. R/L-HT. 


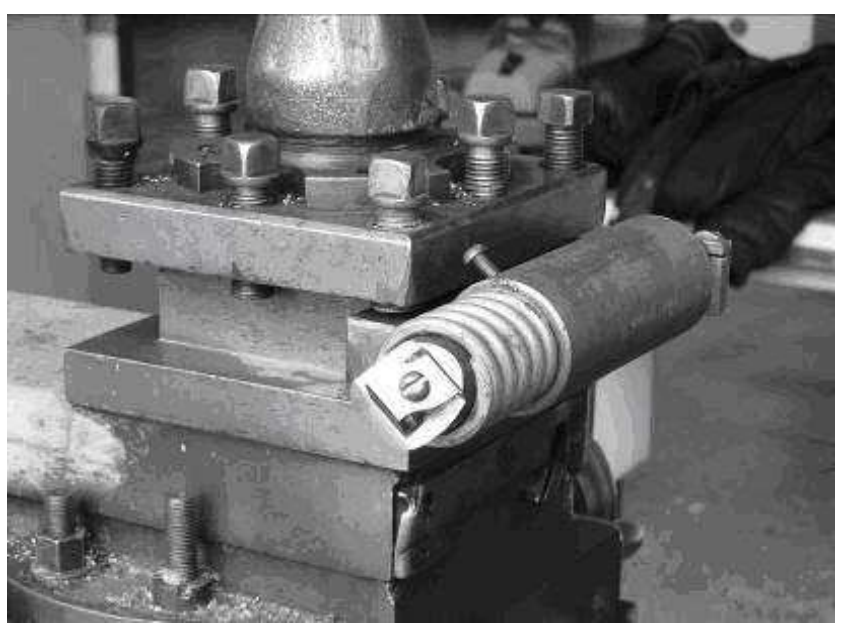

Figure 4. The burnisher.

\subsection{The Results}

After the processing superficial hardness has increased on $30 \%$ (before processing $-180 \mathrm{HB}$, after processing -235 $\mathrm{HB}$ ), a quality of processed surfaces (before processing $\mathrm{Ra}=3,2$ a micron, after processing $-\mathrm{Ra}=0,63$ a micron) has considerably improved. Thus, the effect reached with application of the considered way of installation of firmalloyed indenter in burnisher is positive.

\section{SHS Coverings Modern Energy Efficient Technology}

\subsection{The Theory of Self-propagating High-temperature Synthesis}

Self-propagating high-temperature synthesis (SHS) is a process of moving of a wave of chemical reaction on a mix of reagents with formation of the firm end-products, made for the purpose of synthesis of substances of materials. SHS represents a mode of course strong exothermic reactions (burning reaction) in which the thermal emission is localised in a layer and it is transferred from a layer to a layer by a heat transfer. Self-propagating high-temperature synthesis allows to receive refractory powders, refractory connections, powders of intermetallides, pottery and coverings [7].

One of modern methods of increase of operational characteristics of details of the machines, the cutting tool, and the foundry equipment is application of SHS-coverings. This method allows to notice power-intensive chemistrythermal processing (cementation, nitrating, boriding, etc.) on less power-intensive and not less effective superficial hardening of machine tools. This way allows to increase operational characteristics, such as durability, wear resistance, corrosion resistance [6].

\subsection{The Stages of Research Work}

The purpose of present research work is studying of the wearproof coverings putting on by a method of SHSreactions, and their influence on durable properties of machine detail's. The use of processing consisting of 2-3 stages depending on appointment of a detail and demanded mechanical characteristics is offered in modern machinebuilding [6]. Here are stages, which were investigated in the present work:

1) covering copper. Copper coverings protect steel from corrosion only in the event that they haven't pores. Functional copper coverings are applied to protection of a steel against cementation. Very good electro conductivity copper it is widely used in the electrical engineer and electronics. If the big durability and rigidity is required to put a copper layer is not necessary, if good antifriction qualities and adsorption decrease are required, the copper layer is simply irreplaceable.

2) drawing of a covering by the SHS-reaction method. Powders of connections of manganese and aluminium were used. Manganese is the major component of steel. Its application as alloying element promotes increasing of the hardenability to the steel that characterize depth of the tempered zone at thermal processing. Aluminium provides rising of the heat resistance and corrosion firmness of a steel. Aluminium possesses high heat conductivity, firmness to corrosion for the account of fast formation of strong oxide films protecting a surface from the further interaction. Aluminium introduction promotes formation of a protective film and reduction of the sizes of grain.

3) burnishing. This method of electro physical processing provides not only increasing of hardness of a blanket, but also improves quality of a surface of a detail.

\subsection{The Results}

The average hardness of samples increases in 1,5-2 times, the metal structure is fine-grained, the core is plastic, the quality of a surface is $\mathrm{Ra}=1,25-0,7$ microns. Processing of a detail in diameter of $400-600 \mathrm{~mm}$ and $800-1000 \mathrm{~mm}$ is made length less than 90 minutes.

For reception of similar characteristics it is necessary to perform operations of thermal or chemistry-thermal processing with the subsequent processing of a detail by cutting and an abrasive which as a whole last an order of 2000-2500 minutes.

\section{The Cluster Approach to Definition of Areas of Rational Use of New Technological Tools for Processing of Materials by Cutting}

\subsection{The Main Problem of Modern Tools}

Now, about $70 \%$ of the metal-cutting tool make of a firm alloy of various marks. The most part is nonresharpened replaceable many-sided plates (NRP). They are used in practically all kinds of tools (cutters, mills, drills etc.). It is known, that worn out of NRP, loses in volume (in 
comparison with not worn out plate), only 2-4 \%, and practically all NRP are made entirely of a firm alloy. Thus, it turns out, that to a waste there is a large quantity of a firm alloy, and it basically consists of rare and expensive materials (carbides of tungsten, the titan, etc.). Certainly, the worn out plates go to processing, but it is difficult and expensive process [10].

The sharp increase in the market of quantity of marks of tool materials has complicated a problem of their choice in concrete industrial conditions. Errors in a tool choice lead out to decrease the level of reliability of the cutting tool and the raise of expense of expensive tool materials.

Identification of areas of rational application of each of offered plates or their groups is complicated in the absence of the effective engineering tool generalizing the recommendations about rational application of marks of tool materials taking into account the basic conditions of operation of the tool: marks of a processed material, operation, a condition of a surface of preparation, modes of cutting, quality of a received surface.

\subsection{The Cards of Kohonen}

In presented work on a basis cluster the approach realized with the help of self-optimizing cards of Kohonen, the area of rational use of new materials and designs of firm-alloyed plates of cutting tools for turning processing that allows to reduce quantity of the cutting material going for processing owing to a wrong choice of plates and optimum modes of cutting is defined.

\subsection{The Data Processing}

There had been made casual sample of 85 cutting plates with their basic indicators (type, parameters and a plate material, depth of cutting and giving, processing type) of the catalogue offered by firm-manufacturer on plates of turning group $[8,9]$.



Figure 5. Cards of inputs and clusters of Pramet.

By means of Kohonen's cards giving to us real possibility of a visual estimation of the importance of indicators (characteristics) of firms-manufacturers resulted in catalogues, we represent multidimensional space of entrance factors in a two-dimensional kind in which he is convenient enough for analyzing.

Entrance parametres are: X1 - external processing, X2 internal processing, X3 - rough-working, X4 - semiroughworking, X5 - finishing processing, X6 - pig-iron, X7 stainless steel, X8 - steel.

On the basis of entrance parameters cards of inputs and clusters for Pramet $[9,10,11,12,13]$ have been received (figure 5).

Intensity of color in a certain point of a card is defined by data which there have got: cells with the minimum values are represented by black color, cells with the maximum values white.

\subsection{The Results}

For the analysis of data it is used all cards.

Clusters which are located at the left are more preferable to use for external, internal, rough-working of steel. They are partially possible to use for pig-iron and stainless steel processing. The regime part is well worked.

Clusters which are located above in the middle are more preferable to use plates to external fair processing of steel. They are partially possible to apply for semirough turning and pig-iron processing.

Clusters which are located on the center are more preferable to use plates to internal fair processing of a steel and stainless steel. They are partially possible to use for rough and semirough turning.

Clusters which are located in the right top corner are more preferable to use plates to external draught processing of steel. They are partially possible to use at pig-iron and stainless steel processing.

Clusters which are located in the right bottom corner are more preferable to use plates to external fair processing of a steel and stainless steel. They are partially possible to use for rough and semirough turning.

\section{Conclusion}

Practical value of the made work consists of research of possibilities of application of surface-active substances or SHS-coverings, use of electropulse processing with different tools in technological process of manufacture of details. These methods are energy safety and more harmless in comparison with traditional methods and will allow the receiving of positive economic benefit in machine-building manufacture. With the help self-organised cards of Kohonen it is possible to systematize characteristics of modern materials of cutting plates, and to use them as the effective engineering tool generalizing the recommendations about rational application of cutting plates that allows to lower quantity of the cutting material going for processing owing to a wrong choice of plates and modes of cutting. 


\section{References}

[1] Ambrazon A.A. The surface phenomena and surface-active substances: the Directory / A.A.Abramzon, E.D.Schukin. - L: Chemistry, 1984.

[2] Babey J.I. The physical basis of pulse hardening of a steel and pig-iron. - Kiev: Naukova dumka, 1988. - 240c.

[3] Bagmutov V. P, Parshev S.N., Dudkina N.G., Zaharov I.N. The electromechanical processing: technological and physical bases, properties, realisation. - Novosibirsk: the Science, 2003. $-318 \mathrm{c}$

[4] Jakovlev S.A., Zhiganov V. I. EMP on lathes // STIN.2000. № 6.

[5] Markauskas S.S. The electro-mechanical hardening of a surface by the tool with compulsory rotation of a roller//Researches and workings out in the field of hardening and restoration of details of cars by electromechanical processing. - Ulyanovsk, 1999.

[6] Carbide, Nitride and Boride Materials Synthesis and Processing. Ed. Alan W.Weimer, London-Weinheim-New York-Tokyo-Melburne-Madras: Chapman AND Hall, 1997, $671 \mathrm{pp}$.

[7] Corbin, N.D., and McCauley, J.W., Self-Propagating High Temperature Synthesis (SHS): Current Status and Future Prospects, MTL MS 86-1, Watertown, MA, May 1986
[8] Gah V.M. Choose of rational marks of tool materials. //Reliability of the tool and optimisation of technological systems. The collection of proceedings. - Kramatorsk: DGMA, №14, 2003

[9] Klyuev V., 2005, Non-destructive testing, Russia. Ref. / In .. Klyuyev, F.., C2 .. Rumyantsev et al., Ed. In .. Klyuev .- M. Mashinostroenie, ISBN 5-217-03300-2.

[10] http://www.Pramet.com

[11] The use of neural network techniques for condition monitoring of acoustic cutting tool / S.Kovalevsky, E.Tkachenko L.Tyutyunnik, E.Bugaev, P. Dasic // Neuro networked technologies and their applications: Proceedings of the All-Ukrainian scientific conference with international participation. - Kramatorsk: DSEA, 2013. - P. 51-54.

[12] Kovalevsky SV . Use Kohonen maps for Integrated Assessment of cutting properties of abrasive wheels / S. Kovalevsky, A.Yanyushkin, E.Bugayov // Mechanics XXI century. XI All-Russian Scientific Conference with international participation: summary reports. - Bratsk VPO "BrSU", 2012. - S. 177-180.

[13] The use of Kohonen maps for selection of inserts / E.Kovalevskaya L.Tyutyunnik E.Tulupova, D.Lobanov // Mechanics XXI century. XI All-Russian Scientific Conference with international participation: summary reports. - Bratsk VPO "BrSU", 2012. - S. 175-177. 